\title{
A Statistical Comparison of Zonal Mean and Tidal Signatures in FORMOSAT-3/COSMIC and Ground-Based GPS TECs
}

\author{
Loren C. Chang ${ }^{1, *}$, Charles C. H. Lin ${ }^{2}$, and Jann-Yenq Liu ${ }^{1}$ \\ ${ }^{1}$ Institute of Space Science, National Central University, Jhongli, Taiwan \\ ${ }^{2}$ Department of Earth Science, National Cheng Kung University, Tainan, Taiwan
}

Received 17 April 2012, accepted 17 October 2012

\begin{abstract}
Atmospheric tidal components in the ionosphere can reflect either the in-situ generated quiet-time variation in the ionosphere, or vertically propagating tidal components generated through coupling to lower or middle atmosphere phenomena. Frequency-wavenumber tidal decomposition is a valuable tool for isolating the primary tidal components that drive the dynamics of the middle and upper atmosphere, allowing temporal and spatial variability to be quantified in a systematic manner, provided sufficient local time sampling. To date, two commonly used data sources for such tidal studies in the ionosphere are the FORMOSAT-3/COSMIC (F3/C) satellite constellation and ground-based GPS-derived Global Ionosphere Maps (GIMs). In this study, the migrating diurnal and semidiurnal tidal components, the nonmigrating diurnal eastward 3 (DE3) component, as well as the zonal mean component that dominate quiet-time ionospheric variability are extracted from 2008 F3/C and GIM Total Electron Content (TEC) data, using integration times of 20 days. We find that the zonal mean and tidal TEC components in F3/C and ground-based GIM data show qualitatively similar seasonal variability and spatial structure. However, the maximum amplitudes of the zonal mean and migrating tidal components computed from F3/C are consistently smaller than those from the ground-based GIMs, revealing a systematic difference between the two datasets. Conversely, the DE3 amplitudes are generally larger in F3/C compared to GIM, potentially due to the higher zonal wavenumber of that component.
\end{abstract}

Key words: Atmospheric tides, Ionosphere, TEC, GIM, Validation, COSMIC

Citation: Chang, L. C., C. C.H.Lin, and J.Y. Liu, 2013: A statistical comparison of zonal mean and tidal signatures in FORMOSAT-3/COSMIC and groundbased GPS TECs. Terr. Atmos. Ocean. Sci., 24, 253-263, doi: 10.3319/TAO.2012.10.17.01(SEC)

\section{INTRODUCTION}

Atmospheric tides are the dominant dynamic feature of the Earth's mesosphere and lower thermosphere (MLT), and are defined as global scale oscillations with periods that are harmonics of a solar day. The zonal phase velocity of a tidal component may be calculated based upon its period and zonal wavenumber, which determines the direction and speed at which that tidal component propagates zonally around the Earth. Atmospheric tides are further subdivided based upon their phase velocities into migrating tidal components, which are Sun-synchronous, propagating westward at the same apparent rate as the Sun and nonmigrating tides, which correspond to all other phase velocities, both eastward and westward. Persistent atmospheric tidal components in

\footnotetext{
* Corresponding author

E-mail:loren@jupiter.ss.ncu.edu.tw
}

the MLT correspond to disturbances driven by latent heat release and periodic solar IR water vapor forcing in the troposphere, as well as UV ozone forcing in the stratosphere (Hagan 1996). Other more transient tidal components may arise through nonlinear interaction between the persistent migrating tides and stationary planetary waves (Chang et al. 2009). These tidal disturbances grow in amplitude as they propagate upwards due to the decreasing density of the background atmosphere, attaining maximum amplitude between roughly 90 - $120 \mathrm{~km}$ altitude, before becoming dissipated by turbulent and molecular diffusion in the lower thermosphere (Hagan 1996; Ortland and Alexander 2006; Zhang et al. 2006).

Prior to being dissipated, atmospheric tides that penetrate the lower thermosphere may interact with the ionospheric E-region dynamo to modulate plasma uplift (Zhang et al. 2006). This results in the frequency and wavenumber 
of the interacting tidal component being transmitted further upwards into the ionospheric F region (England et al. 2006). Ionospheric variability resulting from this tidal coupling has been observed in the case of some prominent non-migrating (non-Sun synchronous) tidal components, which are more easily distinguished from structures resulting from the normal quiet-time variation of the ionosphere. Such non-migrating tides include DE3 (the non-migrating diurnal tide with eastward zonal wavenumber 3) (Immel et al. 2006; Lin et al. 2007), and SW1 (the non-migrating semidiurnal tide with westward zonal wavenumber 1) (Pedatella and Forbes 2010; Lin et al. 2012).

Although many early studies of tides in the ionosphere utilized the constant local time analysis commonly used in ionospheric studies (e.g., Immel et al. 2006; Lin et al. 2007; Jee et al. 2010), this method is limited in that the amplitudes and phases of nonmigrating tidal components are aliased to appear as stationary planetary waves, while the Sun-synchronous migrating tides are aliased with zonal mean values (Forbes et al. 2006). To resolve this ambiguity, more recent studies have adopted for the ionosphere the frequency-wavenumber decomposition method commonly used in middle atmospheric tidal studies. In frequency-wavenumber analysis, data across multiple longitudes and times are fit to sinusoidal functions of longitude and time, allowing the amplitude and phase of a specific tidal component (that is, a frequency/zonal wavenumber pair) to be isolated. This method has been applied in recent years to examining well known phenomena in the ionosphere that are local time and longitude dependent, including those resulting from the upwards coupling of middle atmospheric tides, as well as phenomena forced in-situ in the ionosphere.

Results from such frequency-wavenumber tidal decomposition have found that along with the zonal mean field, the migrating tidal components comprise most of the normal quiet-time variation in the ionosphere, including diurnal plasma uplift from the equatorial fountain to form the equatorial ionization anomalies (EIAs), as well as that resulting from in-situ photoionization (Mukhtarov and Pancheva 2011; Lin et al. 2012). In addition to the aforementioned sources, migrating ionospheric tidal components can also reflect changes arising from migrating tides in the middle and lower atmosphere, making the contribution of in-situ ionospheric migrating tidal sources difficult to separate from lower atmospheric sources (Mukhtarov and Pancheva 2011). Nonetheless, migrating tidal modulation by nonlinearly-interacting propagating planetary waves has been suggested as a possible medium for transmitting multi-day planetary wave periodicities into the ionosphere (Pancheva et al. 2006). Recent observations have also shown that short-term variation in ionospheric fields during stratospheric sudden warmings (SSWs) can be attributed to changes in the amplitudes and phases of the migrating ionospheric tidal components (Fang et al. 2012; Lin et al. 2012).
It has also been suggested that a portion of the migrating ionospheric tidal variability during SSW time periods can be attributed to changes in the stratospheric ozone source regions for the migrating tides propagating upwards from the middle atmosphere (Goncharenko et al. 2012). Due to their large amplitudes, changes in the migrating ionospheric tides will have a disproportionate effect on the overall variation of the ionosphere, compared to nonmigrating tides (Lin et al. 2012).

Because of their short periods, global scale observations of atmospheric tides are difficult to obtain, requiring sampling over 24 hours of solar local time at all latitude and longitude zones in order to unambiguously resolve using frequency-wavenumber tidal decomposition (Zhang et al. 2006). At present, several global scale studies of tides in the ionosphere where such decomposition is performed have relied on two sources: Global Ionospheric Maps (GIMs) of Total Electron Content (TEC) derived from ground-based GPS observation networks, and spaceborne observations obtained via GPS occultations from the FORMOSAT-3/COSMIC (F3/C) microsatellite constellation. With the growing application of the frequency-wavenumber tidal decomposition method commonly employed in middle atmospheric tidal studies to those in the ionosphere, as well as the interest in short-term tidal variability, a need exists for comparison of ionospheric tides between these two datasets retrieved using this method. In the following study, we present a comparison of the zonal mean, migrating diurnal (DW1), migrating semidiurnal (SW2), and DE3 tidal TEC components from these two datasets during 2008. The spatial structure, seasonal variation, and systematic differences between the two datasets are shown and discussed.

\section{METHODOLOGY}

The F3/C constellation is comprised of 6 microsatellites launched in April 2006, and provides global observations of the ionosphere with unprecedented spatial scale and temporal resolution. The 6 microsatellites have since been separated into individual orbital planes at roughly $800 \mathrm{~km}$ altitude, $72^{\circ}$ inclination, and $30^{\circ}$ longitudinal separation between adjacent orbital planes. Over 2500 GPS occultations are performed each day, providing electron density profiles up to $800 \mathrm{~km}$ altitude (Cheng et al. 2006; Lin et al. 2007) with 24 hour solar local time coverage each day. For this study, the TEC corresponding to each occultation was computed by integrating electron densities with respect to height, between $200-800 \mathrm{~km}$. Although F3/C TECs will not include contributions above $800 \mathrm{~km}$, past ionospheric tidal structure studies by Mukhtarov and Pancheva (2011) have found that the migrating diurnal, semidiurnal, and DE3 tides examined here all peak between roughly $300-500 \mathrm{~km}$ altitude. Similar to the methodology of Lin et al. (2012), occultations containing negative electron densities or with 
values exceeding $2 \times 10^{6} \mathrm{~cm}^{-3}$ were deemed erroneous and discarded. Utilizing a 20 day sliding window, a latitude/longitude grid of $5^{\circ} \times 5^{\circ}$, and following the linear least-squares fitting method described by Wu et al. (1995), the F3/C TECs were fit to a basis function of the form:

$$
F(\lambda, t)=\bar{F}+\sum_{n=1}^{2} \sum_{s=-4}^{4} \hat{F}_{s, n} \cos \left(n \Omega t-s \lambda-\hat{\psi}_{s, n}\right)
$$

Here $\bar{F}$ is the zonal mean TEC, $s$ is the zonal wavenumber (westward negative), $\Omega$ is the Earth's rotation rate, while $\hat{F}_{s, n}$ and $\hat{\psi}_{s, n}$ are the amplitude and phase of each tidal component, respectively. For this study focusing on the quiet-time variation of the ionosphere, we restrict our analysis to the low and mid-latitudes equator wards of $50^{\circ}$ latitude, where the effects of geomagnetic storms are less pronounced.

Global Ionospheric Maps of TEC are provided via the International GNSS Service (IGS), and produced by the Center for Orbit Determination in Europe (CODE) (Schaer 1999; Hernández-Pajares et al. 2009). Such GIMs are created via assimilation of data from a worldwide network of about 200 GPS/GLONASS receivers, creating a map modeled in a solar-geomagnetic reference frame using a spherical harmonics expansion up to degree and order 15. The resulting GIMs provide global snapshots of TEC with a geographic latitude/longitude resolution of $2.5^{\circ} \times 5^{\circ}$ every 2 hours. Although the irregular distribution of GPS receivers necessitates a smoothing process, the spatial and temporal TEC variations in the GIMs were determined to be in good agreement with observations. Comparing CODE GIM TECs to TOPEX/Jason observations at constant local times, Jee et al. (2010) found that the GIMs reproduced most seasonal, spatial, and local time variability, although certain features such as the EIAs, the Weddel Sea Anomaly, and the wave-4 EIA longitudinal modulation were not well resolved. In our study, the zonal mean, diurnal, and semidiurnal tidal components in the GIM TECs were retrieved using the same methodology previously detailed for the F3/C TEC dataset.

\section{RESULTS}

Figure 1 shows the amplitudes of the computed zonal mean TEC values throughout 2008 as a function of magnetic latitude and time from GIM (Fig. 1a) and COSMIC (Fig. 1b). The zonal mean TEC represents the daily baseline ionization value, and clearly reflects the seasonal variation in the solar zenith angle: the latitude of peak zonal mean TEC amplitude follows the Sun to roughly $+/-15^{\circ}$ latitude in the summer hemisphere. The zonal mean TECs maximize around the equinoxes (days 90 and 300), and minimize around the solstices (days 30 and 210). This variation in zonal mean TEC amplitude corresponds to the known semiannual variation in the ionosphere that has been con- nected to seasonal variations in the $\mathrm{O} / \mathrm{N}_{2}$ ratio (Rishbeth et al. 2000). The twin bands of the EIAs on either side of the magnetic equator are not distinct in the case of the zonal mean TECs, likely due to the diurnal nature of the equatorial fountain mechanism (Appleton 1946). The above characteristics indicate that the zonal mean TECs reflect the constant level of photoionization throughout the ionosphere.

It is apparent from Fig. 1 that the amplitudes of the zonal mean TECs are smaller in $\mathrm{F} 3 / \mathrm{C}$ with maximum values of approximately $13 \mathrm{TECu}$, compared to those in GIM, which attain maximum values near $22 \mathrm{TECu}$. Figure 1c shows the difference between the GIM and F3/C zonal mean TECs as a function of latitude and time. As can be seen from the figure, the zonal mean difference fields are proportionate to the latitudinal and temporal structure of the zonal mean TECs, indicating a relatively constant linear relation between the two datasets. A more quantitative method of visualizing this difference is presented in Fig. 2, which shows a scatterplot of F3/C zonal mean TEC values in 2008, plotted with respect to GIM values from identical times and magnetic latitudes. If the zonal mean TECs from the two datasets were completely identical, all the plotted points would lie along the solid black line with slope equal to 1 . The slope between the actual data points computed via linear regression (red line), denotes the overall bias of the amplitudes of F3/C with respect to GIM. A slope less than (exceeding) 1 indicates that the amplitudes of F3/C are consistently smaller (larger) than those in GIM. In the case of the zonal mean TECs, the slope is determined to be 0.6 , indicating that on average, the F3/C zonal mean TECs are slightly less than $2 / 3 \mathrm{~s}$ of those in GIM. The roughly linear point distribution also yields useful information, indicating that by and large, the spatial structure and variation of the two datasets are consistent, with the relative RMS error being $11 \%$.

We now examine results for the migrating diurnal tide (24 hour period, zonal wavenumber westward 1, DW1) in TEC, shown as a function of magnetic latitude and time in Fig. 3. Similar to the zonal mean results shown previously, the migrating diurnal tide shows strong dependence on the seasonal variation in solar zenith angle. The latitudinal structure also maximizes in the region under the solar zenith point, although the twin peaks of the EIAs about the magnetic equator are resolved in the GIM results, particularly in the two months surrounding the March equinox. The semiannual TEC amplitude variation in DW1 may also be related to that in the background ionospheric electron density, as in the case of the zonal mean values. However, given the large amplitudes of the migrating diurnal tide in the equatorial $\mathrm{E}$ region, we cannot exclude the possibility that this also reflects upwards coupling of the semiannual variation of DW1 in the MLT region, which has been attributed to seasonal changes in both solar heating and the middle atmospheric zonal mean zonal winds (McLandress 2002a, b). The phases (not shown) in both datasets show that the migrating diurnal 
tide maximizes around 14 hours solar local time at all locations throughout the mid and low latitude area. These results are again indicative that the migrating diurnal tide in TEC reflects photoionization, consistent with the observations of Mukhtarov and Pancheva (2011) in F3/C electron density.

The amplitudes of the migrating diurnal tide are comparable though slightly smaller than those of the zonal mean TEC. F3/C shows maximum amplitudes of $12 \mathrm{TECu}$, which is again smaller than the $18 \mathrm{TECu}$ in GIM. The slope computed from the scatterplots of the migrating diurnal tide shown in Fig. 4 shows a value of 0.7, again reflecting the constantly smaller F3/C amplitudes. The distribution of the data points in the scatterplot again exhibit a mostly linear relation, illustrating the similarity in spatial and seasonal variation, with an RMS error of $10.6 \%$ between the two datasets.

Figure 5 shows the amplitudes of the migrating semidiurnal tide (SW2) in TECs as a function of latitude and time. Compared to the zonal mean and migrating diurnal tide, the migrating semidiurnal tide in TECs exhibits a clear bimodal
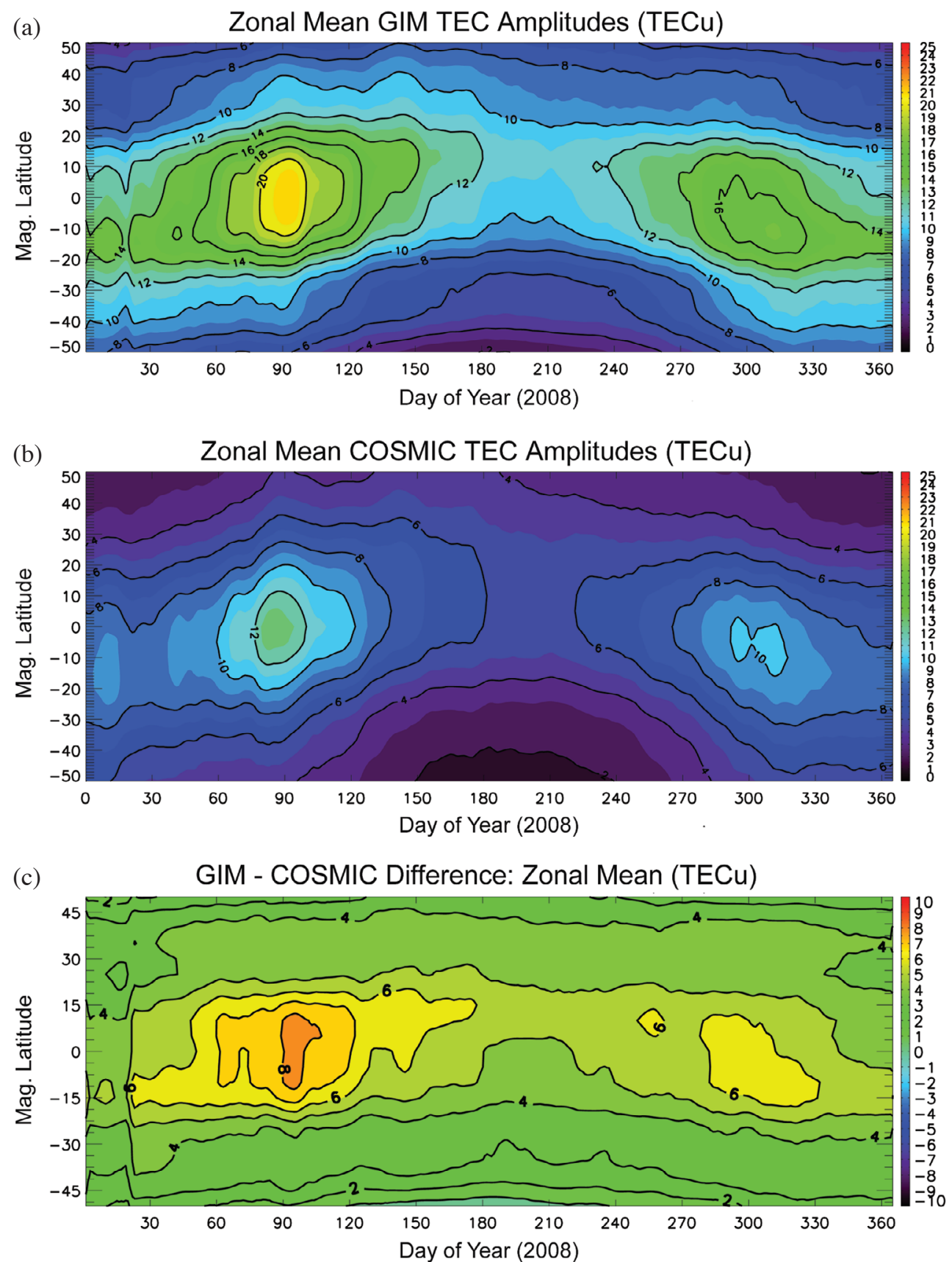

Fig. 1. Zonal mean amplitudes computed from GIM (a) and COSMIC (b) TECs as a function of magnetic latitude and time for 2008, contours of 2 $\mathrm{TECu}$; (c) zonal mean amplitude difference between COSMIC and GIM, contours of 1 TECu. 


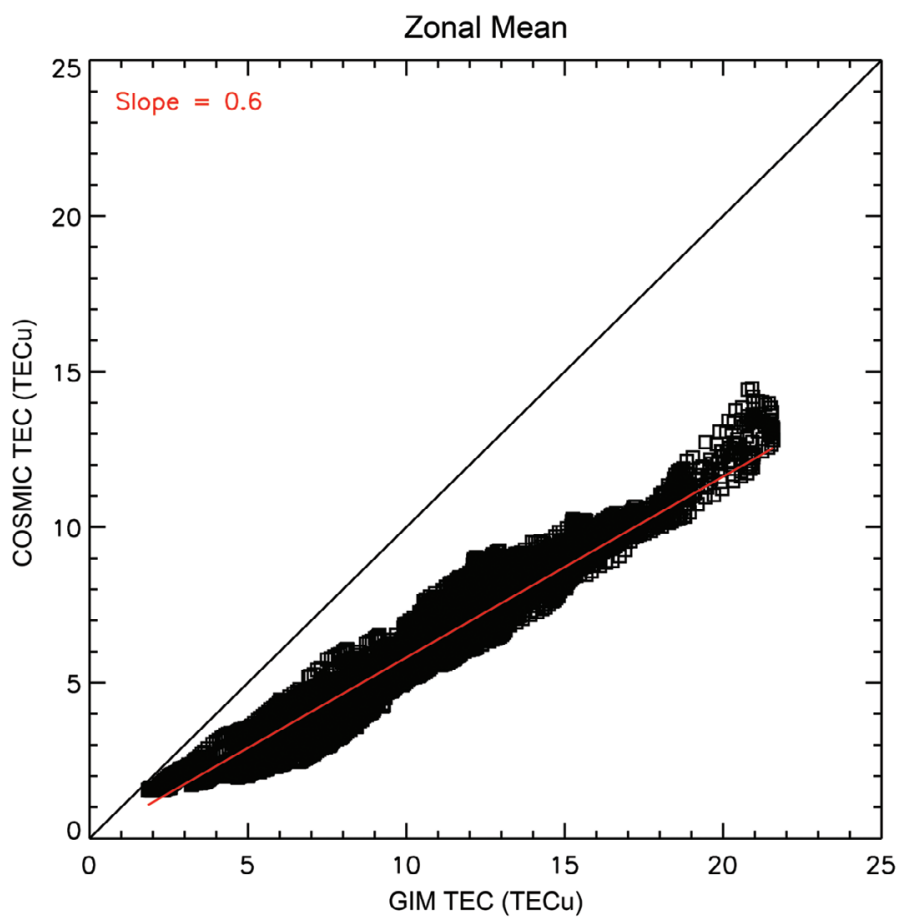

Fig. 2. Scatterplot of zonal mean TECs from COSMIC plotted with respect to values from identical times and latitudes in GIM. Solid black line denotes line where slope $=1$. Red line indicates slope of best fit with slope $=0.6$.
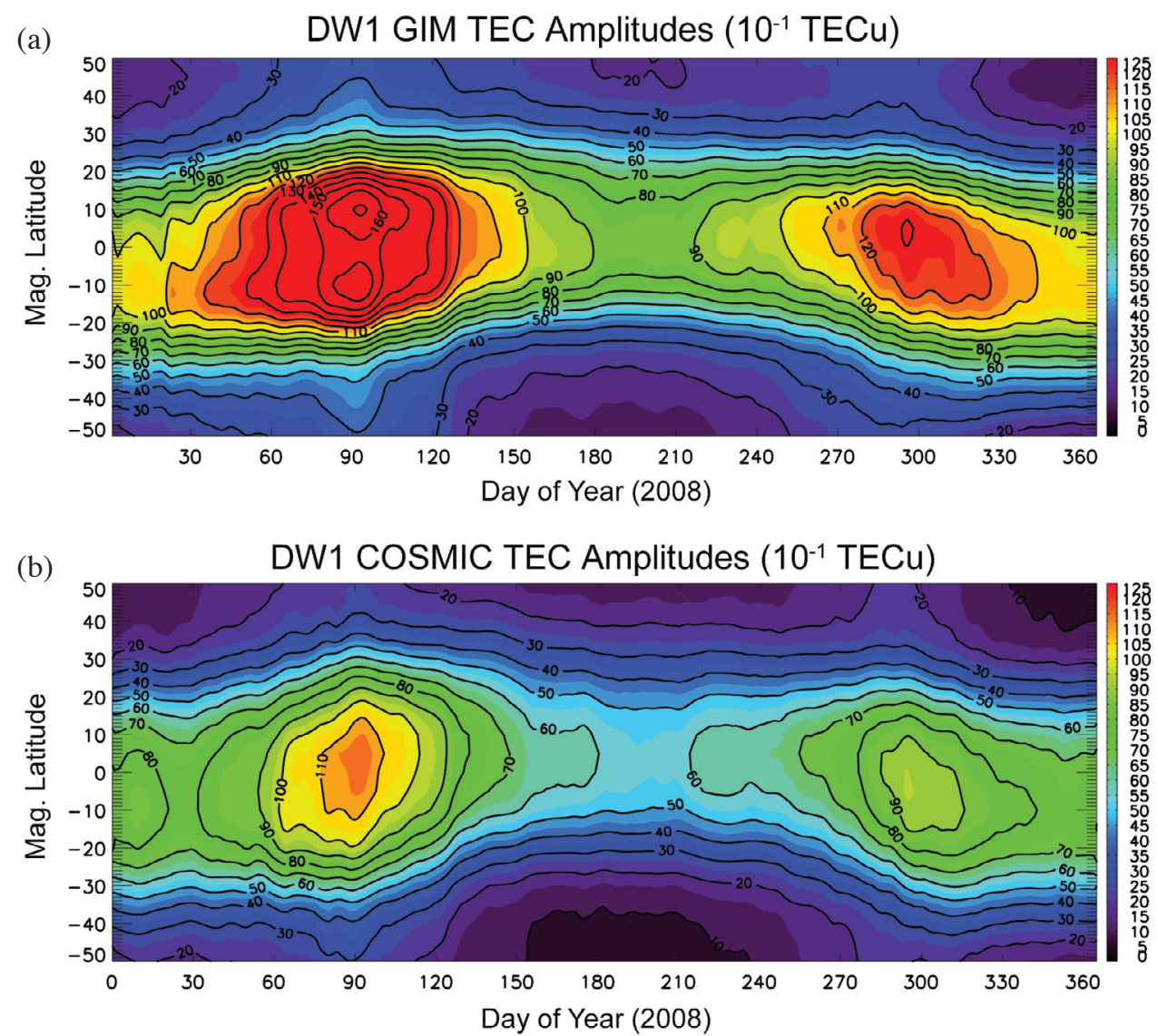

Fig. 3. Migrating diurnal tide amplitudes computed from GIM (a) and COSMIC (b) TECs as a function of magnetic latitude and time for 2008. Contours of $10 \times 10^{-1} \mathrm{TECu}$. 


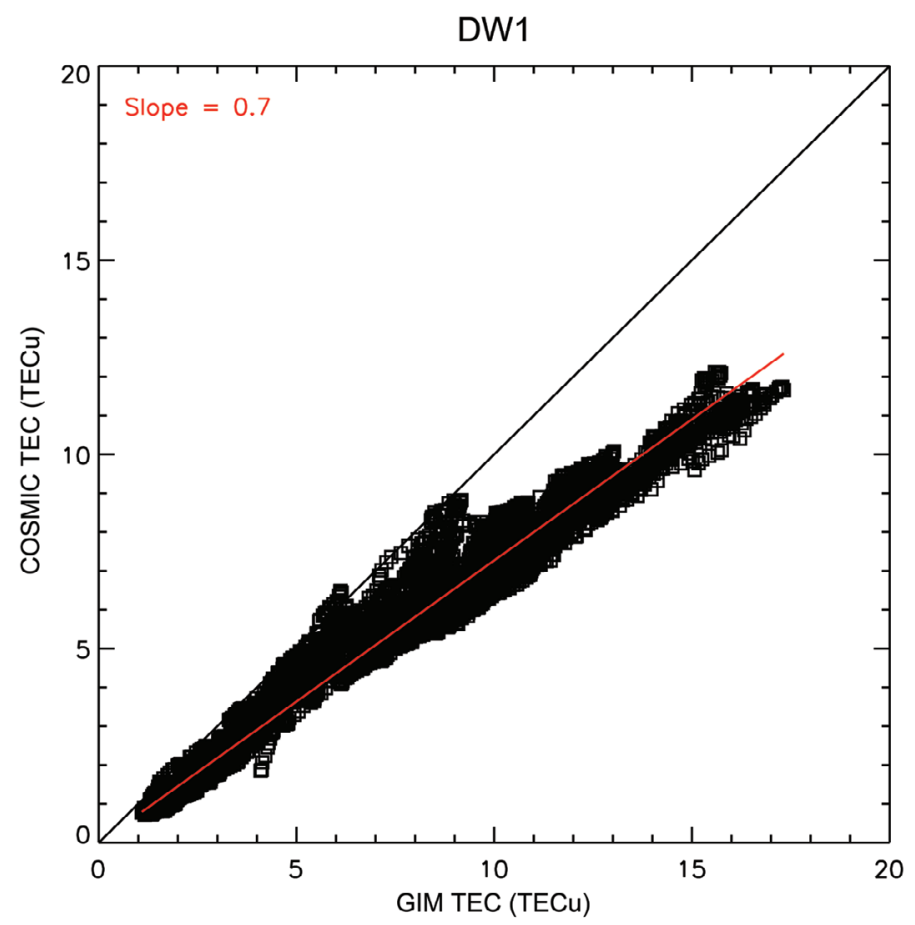

Fig. 4. Scatterplot of migrating diurnal tide TECs from COSMIC plotted with respect to values from identical times and latitudes in GIM. Solid black line denotes line where slope $=1$. Red line indicates slope of best fit with slope $=0.7$.
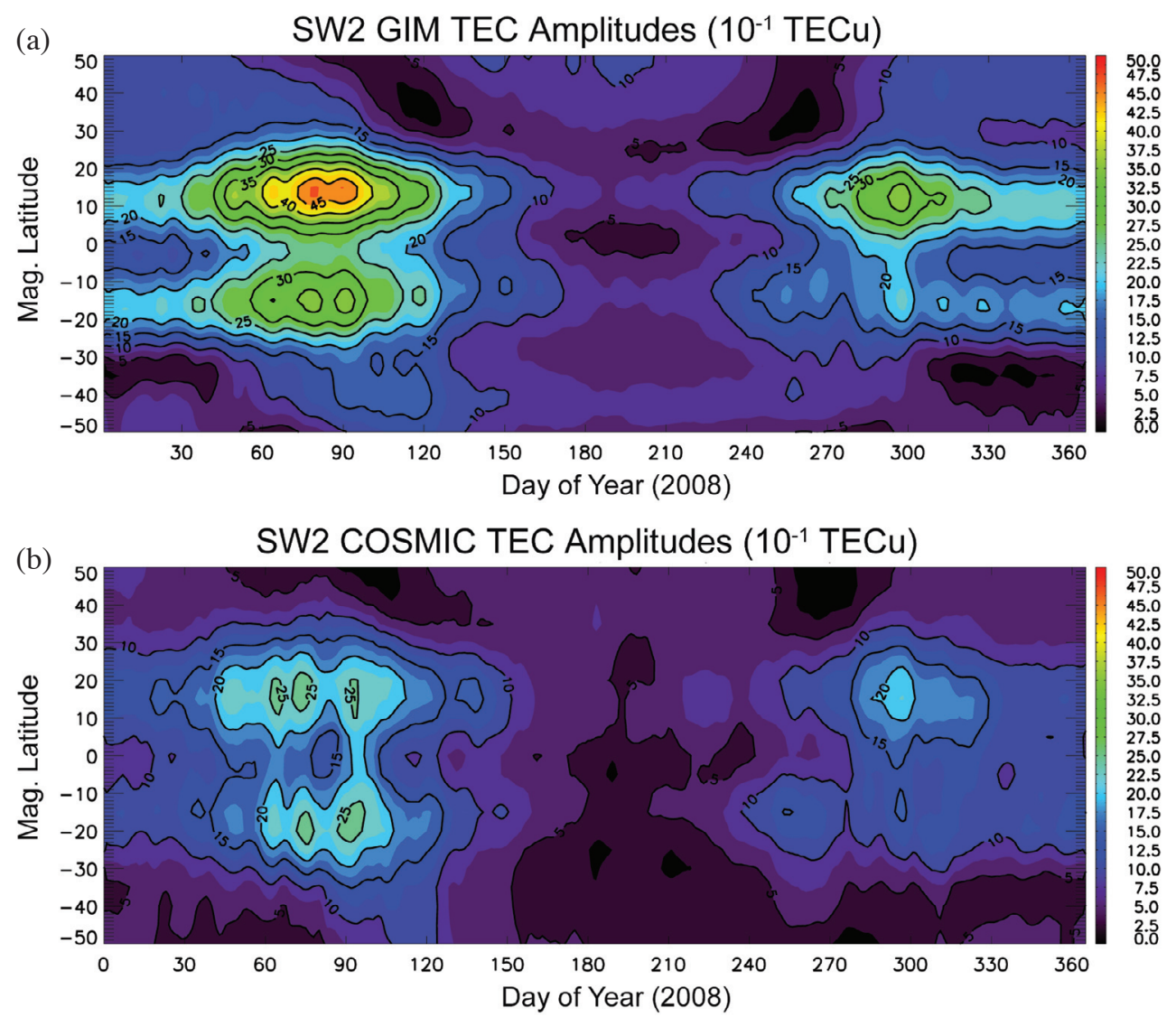

Fig. 5. Migrating semidiurnal tide amplitudes computed from GIM (a) and COSMIC (b) TECs as a function of magnetic latitude and time for 2008. Contours of $5 \times 10^{-1} \mathrm{TECu}$. 
structure in latitude, with peaks located roughly at $15^{\circ}$ latitude in both hemispheres, consistent with the location of the EIAs. Mukhtarov and Pancheva (2011) suggested that a similar bimodal distribution observed in electron densities near $400 \mathrm{~km}$ is indicative of upwards coupling of the migrating semidiurnal tide in the MLT region via the equatorial fountain mechanism. The seasonal variation is again semiannual in nature with maxima (minima) in the postequinox (post-solstice) periods at the same times as those in the zonal mean and migrating diurnal tide. Unlike the aforementioned components however, the migrating semidiurnal tidal amplitudes in TEC do not show any apparent dependence on solar zenith angle, and show little seasonal latitudinal shift in the locations of amplitude maxima. The phases (not shown) are constant around the equinoxes, but do show a 4 to 6 hour shift in the mid to high latitudes of the summer hemisphere. However, given the small amplitudes of the migrating semidiurnal tide during the solstices, this phase shift does not appear to be significant.

The scatterplot for the migrating semidiurnal tide shown in Fig. 6 also reflects consistency in spatial and temporal variation between the two datasets, as well as the persistent underestimation of tidal amplitudes by $\mathrm{F} 3 / \mathrm{C}$ relative to GIM. The slope is 0.7 , and the RMS error is $28.9 \%$. This is again similar to the results for the zonal mean and migrating diurnal tides, though the higher relative error reflects the generally lower amplitudes compared to the zonal mean and migrating diurnal components. The maximum amplitude of the migrating semidiurnal tide is $3 \mathrm{TECu}$ in $\mathrm{F} 3 / \mathrm{C}$, and $5 \mathrm{TECu}$ in GIM, which corresponds to roughly $20-25 \%$ of the amplitudes of the zonal mean and migrating diurnal tides in TEC.

Figure 7 shows the amplitudes of the DE3 non-migrating diurnal tide as a function of latitude and time. The seasonal variation of DE3 is similar in both datasets, and correlates well with the known behavior of DE3 in the ionosphere (Wu et al. 2009; Mukhtarov and Pancheva 2011) and thermospheric neutral zonal winds (Häusler and Lühr 2009), exhibiting two distinct peaks throughout the course of the year in March/April and September/October, with smaller sporadic enhancements at other times. The seasonal variation of DE3 in TECs is likely due to the variation of DE3 in the MLT region, which exhibits a primary peak in September/October, a secondary peak in March/April, and a smaller peak in January (Wu et al. 2009; Pancheva et al. 2010; Mukhtarov and Pancheva 2011). This has also been attributed in part, to the aforementioned semiannual variation in background ionospheric electron densities, which may feedback into any tidal modulation of vertical plasma drift (Mukhtarov and Pancheva 2011). This possibility is further elucidated in Fig. 8, which shows the daily latitude variation of DE3 amplitudes normalized by the maximum equatorial zonal mean TEC amplitude for each day. The normalized DE3 amplitudes still maximize in September/October, but the March/April peak is significantly reduced, owing to the larger zonal mean TEC amplitudes during this time.

The DE3 amplitudes are concentrated in two bands near EIA latitudes, consistent with its role in generating the well-known wave 4 pattern resolved in the EIAs when viewed in a constant local time reference frame (Immel et al. 2006). However, the DE3 enhancements in F3/C extend more broadly in latitude compared to GIM. Unlike the migrating tides and zonal mean, the amplitudes of DE3 are generally smaller in GIM compared to F3/C, although the GIM September/October peak is slightly larger than F3/C in the southern EIA region. DE3 attains maximum amplitudes of 1.4 TECu in F3/C and 1.2 TECu in GIM. From the scatterplot in Fig. 9, the slope is found to be 1.2, indicating that the DE3 amplitudes in $\mathrm{F} 3 / \mathrm{C}$ are roughly $20 \%$ larger than those in GIM. The RMS is $58.3 \%$, reflecting a somewhat larger degree of spatial difference between the two datasets, as well as the smaller amplitudes. These discrepancies may be related to the shorter wavelength (larger zonal wavenumber) of DE3 compared to the aforementioned migrating tides. We expect that the denser sampling offered by F3/C will result in better resolution of DE3. In contrast, GIM must rely on assimilation of a sparser network of irregularly spaced GPS receivers, resulting in a smoothing effect, which will be more pronounced for higher zonal wavenumber components.

We also note that Jee et al. (2010) found in their comparisons between GIM and TOPEX/Jason TECs that the longitudinal wave-4 structure, of which DE3 is a major contributor, was not resolved in constant local time plots of GIM. The results shown here indicate that DE3 is indeed present in our revision of GIM, albeit at amplitudes smaller than those observed by F3/C. This may be the result of different GIM revisions, and also reflects the signal isolation capabilities of the least-squares fitting algorithm used in our study compared to constant local time analysis.

\section{DISCUSSION AND CONCLUSION}

We have presented the results of a statistical comparison between the zonal mean, migrating diurnal, migrating semidiurnal, and DE3 non-migrating tides in TECs from the F3/C and GIM datasets in 2008. Together, these three components comprise a considerable portion of ionospheric variability (Lin et al. 2012), as well as the well-known wave 4 EIA structure (Immel et al. 2006). Our results show that both F3/C and GIM show spatial structure and seasonal variation for the aforementioned components that are consistent with each other, as evidenced by their relatively linear distribution when plotted with respect to one another, and are also in agreement with previous studies.

The zonal mean and migrating diurnal tides in TEC show a clear dependence on the solar zenith angle, undergoing a semiannual variation consistent with generation via 


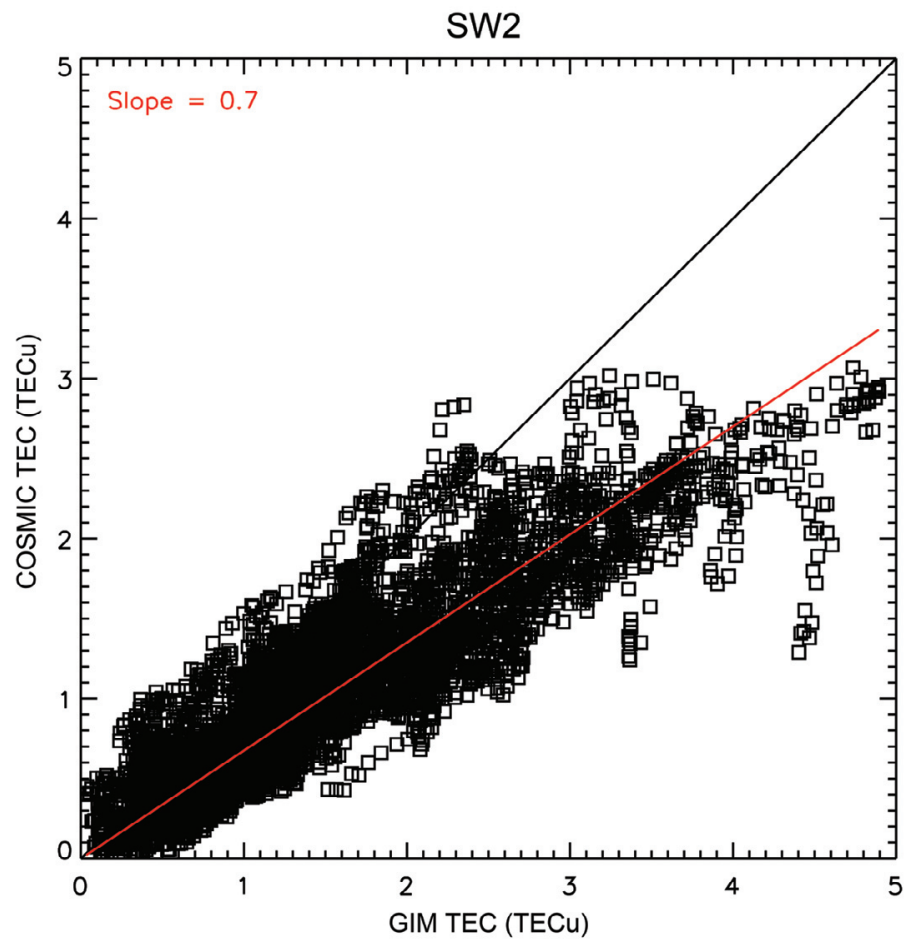

Fig. 6. Scatterplot of migrating semidiurnal tide TECs from COSMIC plotted with respect to values from identical times and latitudes in GIM. Solid black line denotes line where slope $=1$. Red line indicates slope of best fit with slope $=0.7$.
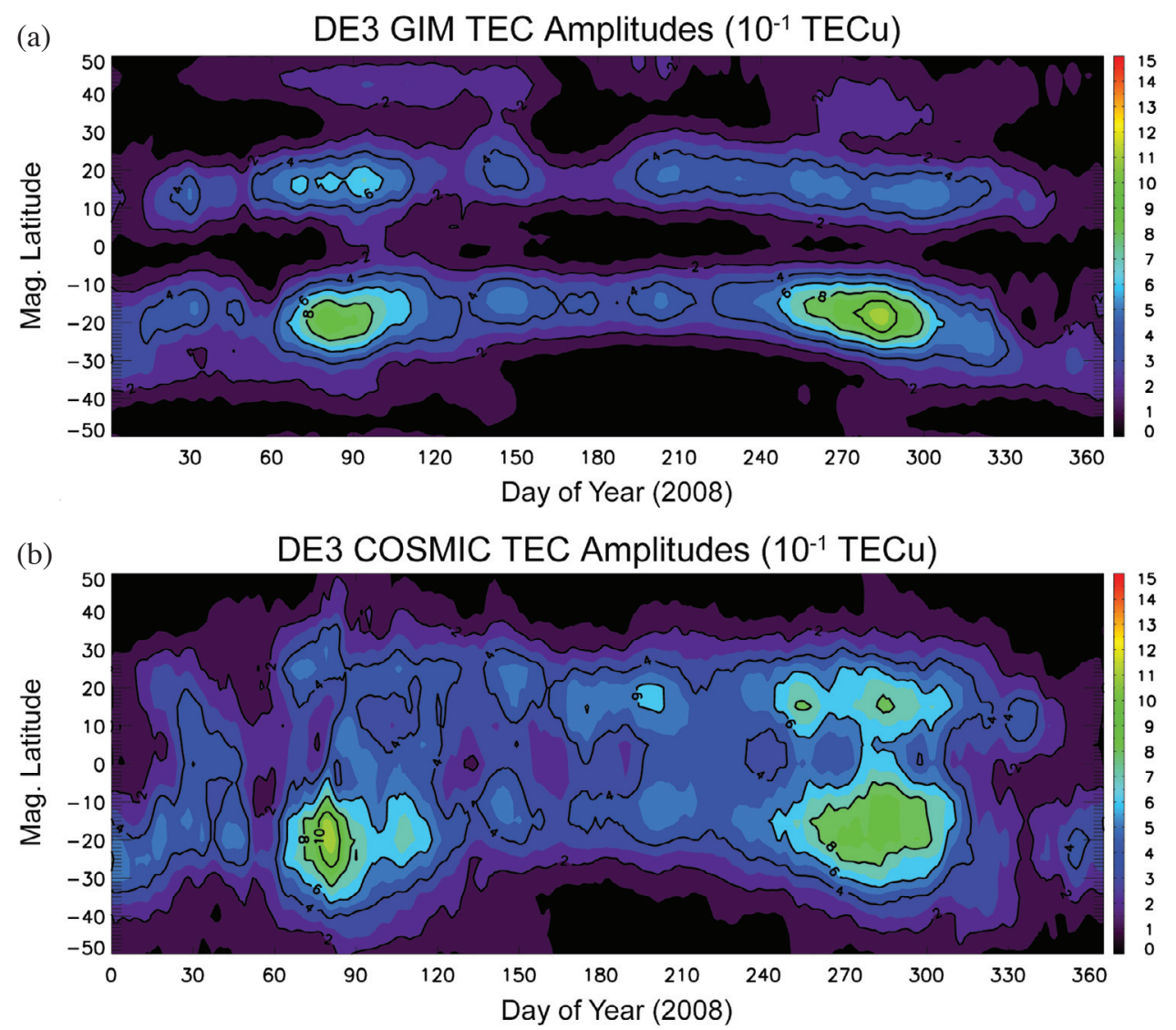

Fig. 7. DE3 Non-migrating diurnal tide amplitudes computed from GIM (a) and COSMIC (b) TECs as a function of magnetic latitude and time for 2008. Contours of $2 \times 10^{-1} \mathrm{TECu}$ 

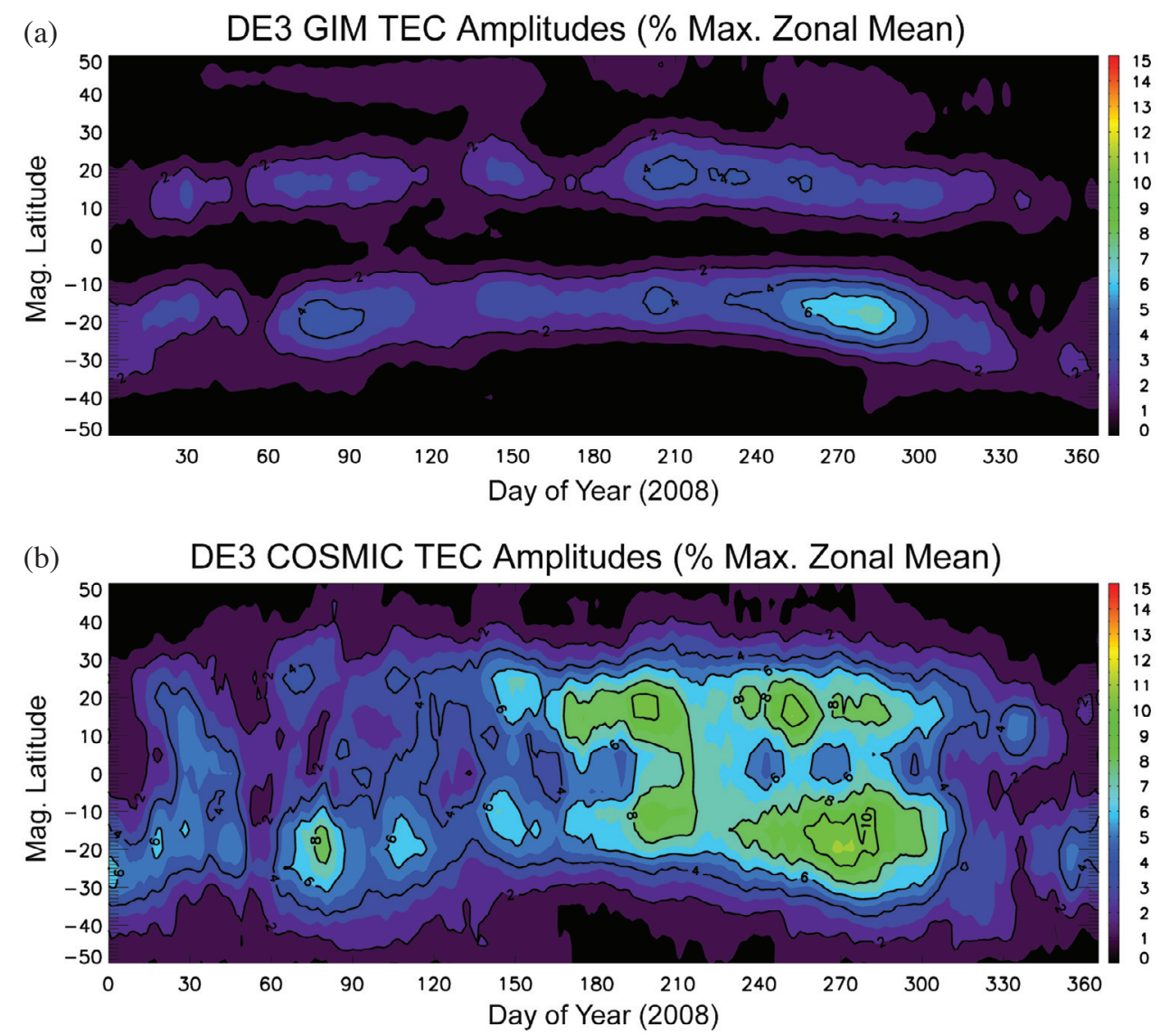

Fig. 8. DE3 Non-migrating diurnal tide amplitudes normalized by the maximum equatorial zonal mean TEC amplitude from each day, computed from GIM (a) and COSMIC (b) TECs as a function of magnetic latitude and time for 2008. Contours of $2 \%$.

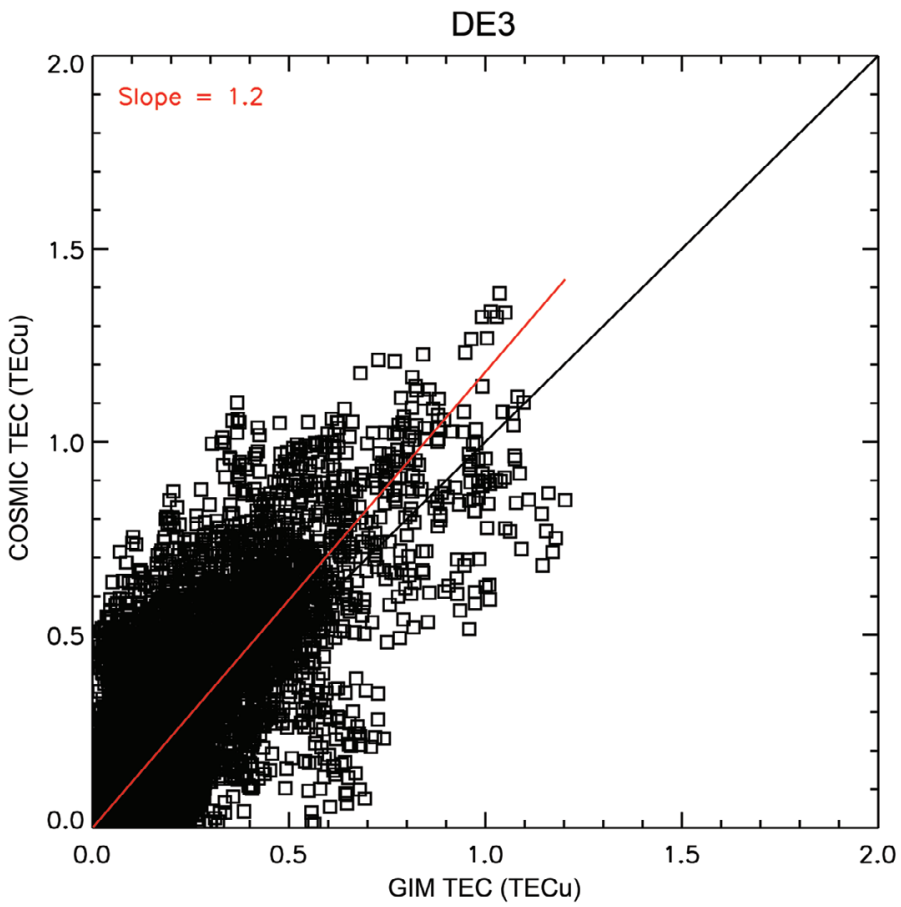

Fig. 9. Scatterplot of DE3 TECs from COSMIC plotted with respect to values from identical times and latitudes in GIM. Solid black line denotes line where slope $=1$. Red line indicates slope of best fit with slope $=1.2$. 
photoionization. It is also interesting to note that the January amplitude minima in both of these components corresponds to the time period around SSW events, indicating that future work will have to be performed to understand the extent to which seasonal variation influences ionospheric variability during this time (Lin et al. 2012).

The migrating semidiurnal tide is only about $20-25 \%$ of the amplitudes of the two aforementioned components, and shows strong bimodal structure in latitude previously attributed by Mukhtarov and Pancheva (2011) to generation through modulation of the equatorial fountain mechanism by the migrating semidiurnal tide in the E-region.

Despite the similarities in spatial and temporal variation, the zonal mean and migrating tidal amplitudes computed from the two datasets show prominent differences in amplitude, with amplitudes from F3/C consistently smaller than those from GIM by roughly a factor of $1 / 3$. Potential explanations for this systematic discrepancy may include differences in the inversion processes utilized for $\mathrm{F} 3 / \mathrm{C}$, post processing criteria utilized in the $\mathrm{F} 3 / \mathrm{C}$ tidal fitting process, as well as smoothing from the assimilation process utilized in the construction of the GIMs attributing greater activity to waves and tides of longer zonal wavelengths (smaller zonal wavenumbers). Another factor is that F3/C TECs extend only to the $800 \mathrm{~km}$ orbital altitude, with observations from Pedatella et al. (2011) showing roughly 10 TECu between $800-20200 \mathrm{~km}$ at low latitudes. However, we note that the F3/C observations still cover much of the ionosphere including and above the $\mathrm{F}$ region peak, thus encompassing much of the electron content contributing to the structure of the examined tidal components (Mukhtarov and Pancheva 2011).

The results for DE3 show that the amplitudes of that component are generally smaller in GIM compared to F3/C, and are much narrower in latitude when resolved in the former compared to the latter. This may be related to the shorter zonal wavelength (higher zonal wavenumber) of DE3 compared to the aforementioned migrating tides and zonal mean component. Because of this, it is expected that the sparser network of GPS receivers used to construct the GIM will be less effective at resolving DE3, compared to the denser sampling offered by F3/C.

Acknowledgements This work was supported by funding from the National Science Council of Taiwan under grants NSC 100-2628-M-006-002-MY3 to C. H. Lin, NSC 98-2111-M-008-MY3 to J. Y. Liu, and NSC 101-2111-M008-021-MY2 to L. C. Chang. The authors thank J. T. Lin and J. T. Jhou of NCKU, as well as H. H. Huang and S. Y. Chang of NCU for their advice and support. We also thank NSPO and UCAR for provision of FORMOSAT-3/COSMIC data, and CODE for access to GIM TECs. The first author also thanks colleagues at $2^{\text {nd }}$ Battery, ROC Missile Command $625^{\text {th }}$ Air Defense Artillery Battalion, Hualien for support and inspiration.

\section{REFERENCES}

Appleton, E. V., 1946: Two anomalies in the ionosphere. Nature, 157, 691-691.

Chang, L. C., S. E. Palo, and H. L. Liu, 2009: Short-term variation of the $s=1$ nonmigrating semidiurnal tide during the 2002 stratospheric sudden warming. J. Geophys. Res., 114, D03109, doi: 10.1029/2008JD010886. [Link]

Cheng, C.-Z. F., Y. H. Kuo, R. A. Anthes, and L. Wu, 2006: Satellite constellation monitors global and space weather. Eos, Trans., AGU, 87, 166-166, doi: 10.1029/ 2006EO170003. [Link]

England, S. L., T. J. Immel, E. Sagawa, S. B. Henderson, M. E. Hagan, S. B. Mende, H. U. Frey, C. M. Swenson, and L. J. Paxton, 2006: Effect of atmospheric tides on the morphology of the quiet time, postsunset equatorial ionospheric anomaly. J. Geophys. Res., 111, A10S19, doi: 10.1029/2006JA011795. [Link]

Fang, T. W., T. Fuller-Rowell, R. Akmaev, F. Wu, H. Wang, and D. Anderson, 2012: Longitudinal variation of ionospheric vertical drifts during the 2009 sudden stratospheric warming. J. Geophys. Res., 117, A03324, doi: 10.1029/2011JA017348. [Link]

Forbes, J. M., J. Russell, S. Miyahara, X. Zhang, S. Palo, M. Mlynczak, C. J. Mertens, and M. E. Hagan, 2006: Troposphere-thermosphere tidal coupling as measured by the SABER instrument on TIMED during JulySeptember 2002. J. Geophys. Res., 111, A10S06, doi: 10.1029/2005JA011492. [Link]

Goncharenko, L. P., A. J. Coster, R. A. Plumb, and D. I. V. Domeisen, 2012: The potential role of stratospheric ozone in the stratosphere-ionosphere coupling during stratospheric warmings. Geophys. Res. Lett., 39, L08101, doi: 10.1029/2012GL051261. [Link]

Hagan, M. E., 1996: Comparative effects of migrating solar sources on tidal signatures in the middle and upper atmosphere. J. Geophys. Res., 101, 21213-21222, doi: 10.1029/96JD01374. [Link]

Häusler, K. and H. Lühr, 2009: Nonmigrating tidal signals in the upper thermospheric zonal wind at equatorial latitudes as observed by CHAMP. Ann. Geophys., 27, 2643-2652, doi: 10.5194/angeo-27-2643-2009. [Link]

Hernández-Pajares, M., J. M. Juan, J. Sanz, R. Orus, A. Garcia-Rigo, J. Feltens, A. Komjathy, S. C. Schaer, and A. Krankowski, 2009: The IGS VTEC maps: A reliable source of ionospheric information since 1998. J. Geodesy, 83, 263-275. doi: 10.1007/s00190-008-0266-1. [Link]

Immel, T. J., E. Sagawa, S. L. England, S. B. Henderson, M. E. Hagan, S. B. Mende, H. U. Frey, C. M. Swenson, and L. J. Paxton, 2006: Control of equatorial ionospheric morphology by atmospheric tides. Geophys. Res. Lett., 33, L15108, doi: 10.1029/2006GL026161. [Link] 
Jee, G., H. B. Lee, Y. H. Kim, J. K. Chung, and J. Cho, 2010: Assessment of GPS global ionosphere maps (GIM) by comparison between CODE GIM and TOPEX/Jason TEC data: Ionospheric perspective. J. Geophys. Res., 115, A10319, doi: 10.1029/2010JA015432. [Link]

Lin, C. H., W. Wang, M. E. Hagan, C. C. Hsiao, T. J. Immel, M. L. Hsu, J. Y. Liu, L. J. Paxton, T. W. Fang, and C. H. Liu, 2007: Plausible effect of atmospheric tides on the equatorial ionosphere observed by the FORMOSAT-3/COSMIC: Three-dimensional electron density structures. Geophys. Res. Lett., 34, L11112, doi: 10.1029/2007GL029265. [Link]

Lin, J. T., C. H. Lin, L. C. Chang, H. H. Huang, J. Y. Liu, A. B. Chen, C. H. Chen, and C. H. Liu, 2012: Observational evidence of ionospheric migrating tide modification during the 2009 stratospheric sudden warming. Geophys. Res. Lett., 39, L02101, doi: 10.1029/20 11GL050248. [Link]

McLandress, C., 2002a: The seasonal variation of the propagating diurnal tide in the mesosphere and lower thermosphere. Part I: The role of gravity waves and planetary waves. J. Atmos. Sci., 59, 893-906, doi: 10.1175/ 1520-0469(2002)059<0893:TSVOTP>2.0.CO;2. [Link]

McLandress, C., 2002b: The seasonal variation of the propagating diurnal tide in the mesosphere and lower thermosphere. Part II: The role of tidal heating and zonal mean winds. J. Atmos. Sci., 59, 907-922, doi: 10.1175/ 1520-0469(2002)059<0907:TSVOTP>2.0.CO;2. [Link]

Mukhtarov, P. and D. Pancheva, 2011: Global ionospheric response to nonmigrating DE3 and DE2 tides forced from below. J. Geophys. Res., 116, A05323, doi: 10.10 29/2010JA016099. [Link]

Ortland, D. A. and M. J. Alexander, 2006: Gravity wave influence on the global structure of the diurnal tide in the mesosphere and lower thermosphere. J. Geophys. Res., 111, A10S10, doi: 10.1029/2005JA011467. [Link]

Pancheva, D. V., P. J. Mukhtarov, M. G. Shepherd, N. J. Mitchell, D. C. Fritts, D. M. Riggin, S. J. Franke, P. P. Batista, M. A. Abdu, I. S. Batista, B. R. Clemesha, and T. Kikuchi, 2006: Two-day wave coupling of the low-latitude atmosphere-ionosphere system. J. Geo- phys. Res., 111, A07313, doi: 10.1029/2005JA011562. [Link]

Pancheva, D., P. Mukhtarov, and B. Andonov, 2010: Global structure, seasonal and interannual variability of the eastward propagating tides seen in the SABER/TIMED temperatures (2002-2007). Adv. Space Res., 46, 257274, doi: 10.1016/j.asr.2010.03.026. [Link]

Pedatella, N. M. and J. M. Forbes, 2010: Evidence for stratosphere sudden warming-ionosphere coupling due to vertically propagating tides. Geophys. Res. Lett., 37, L11104, doi: 10.1029/2010GL043560. [Link]

Pedatella, N. M., J. M. Forbes, A. Maute, A. D. Richmond, T.-W. Fang, K. M. Larson, and G. Millward, 2011: Longitudinal variations in the $\mathrm{F}$ region ionosphere and the topside ionosphere-plasmasphere: Observations and model simulations. J. Geophys. Res., 116, A12309, doi: 10.1029/2011JA016600. [Link]

Rishbeth, H., I. C. F. Müller-Wodarg, L. Zou, T. J. FullerRowell, G. H. Millward, R. J. Moffett, D. W. Idenden, and A. D. Aylward, 2000: Annual and semiannual variations in the ionospheric F2-layer: II. Physical discussion. Ann. Geophys., 18, 945-956, doi: 10.1007/s005 85-000-0945-6. [Link]

Schaer, S., 1999: Mapping and predicting the Earth's ionosphere using the Global Positioning System. Ph.D. Dissertation, Astronomical Institute, University of Berne, Berne, Switzerland, 25 March.

Wu, D. L., P. B. Hays, and W. R. Skinner, 1995: A least squares method for spectral analysis of space-time series. J. Atmos. Sci., 52, 3501-3511, doi: 10.1175/15200469(1995)052<3501:ALSMFS>2.0.CO;2. [Link]

Wu, Q., S. C. Solomon, Y. H. Kuo, T. L. Killeen, and J. $\mathrm{Xu}, 2009$ : Spectral analysis of ionospheric electron density and mesospheric neutral wind diurnal nonmigrating tides observed by COSMIC and TIMED satellites. Geophys. Res. Lett., 36, L14102, doi: 10.1029/20 09GL038933. [Link]

Zhang, X., J. M. Forbes, M. E. Hagan, J. M. Russell III, S. E. Palo, C. J. Mertens, and M. G. Mlynczak, 2006: Monthly tidal temperatures $20-120 \mathrm{~km}$ from TIMED/SABER. J. Geophys. Res., 111, A10S08, doi: 10.1029/2005JA011504. [Link] 